\title{
Opioid Reduction and Risk Mitigation in VA Primary Care: Outcomes from the Integrated Pain Team Initiative
}

\author{
Karen H. Seal, MD, $M P H^{1,2}{ }^{\oplus}$, Tessa Rife, PharmD, $B C G P^{1,2}$, Yongmei Li, $P h D^{7}$, \\ Carolyn Gibson, $P h D^{1,2}$, and Jennifer Tighe, $\mathrm{MSPH}^{7}$
}

'San Francisco Veterans Affairs Health Care System, University of California, San Francisco, San Francisco, CA, USA; ${ }^{2}$ Departments of Medicine and Psychiatry, University of California, San Francisco, San Francisco, CA, USA.

BACKGROUND: National guidelines advise decreasing opioids for chronic pain, but there is no guidance on implementation.

OBJECTIVE: To evaluate the effectiveness of an Integrated Pain Team (IPT) clinic in decreasing opioid dose and mitigating opioid risk.

DESIGN:This study prospectively compared two matched cohorts receiving chronic pain care through IPT $(N=147)$ versus usual primary care (UPC, $N=147$ ) over 6 months. Patients were matched on age, sex, psychiatric diagnoses, and baseline opioid dose.

PATIENTS: Veterans receiving care at a VA medical center or VA community-based clinics.

INTERVENTION: Interdisciplinary IPT, consisting of a collocated medical provider, psychologist, and pharmacist embedded in VA primary care providing short-term biopsychosocial management of veterans with chronic pain and problematic opioid use.

MAIN MEASURES: Change in opioid dose expressed as morphine equivalent daily dose (MEDD) and opioid risk mitigation evaluated at baseline, 3 months, and 6 months. KEY RESULTS: Compared with veterans receiving UPC, those followed by IPT had a greater mean MEDD decrease of $42 \mathrm{mg}$ versus $8 \mathrm{mg}$ after 3 months and $56 \mathrm{mg}$ versus $17 \mathrm{mg}$ after 6 months. In adjusted analysis, compared with UPC, veterans in IPT achieved a 34-mg greater mean reduction at 3 months $(p=0.002)$ and 38-mg greater mean reduction at 6 months $(p=0.003)$. Nearly twice as many patients receiving care through IPT versus UPC reduced their daily opioid dose by $\geq 50 \%$, representing more than a two-fold improvement at 3 months, which was sustained at 6 months [odds ratio $=$ 2.03; 95\% CI $=1.04-3.95, p=0.04]$. Significant improvements were also demonstrated in opioid risk mitigation by 6 months, including increased urine drug screen monitoring, naloxone kit distribution, and decreased co-prescription of opioids and benzodiazepines (all $p$ values $<0.001$ ).

CONCLUSIONS: Interdisciplinary biopsychosocial models of pain care can be embedded in primary care and lead to significant improvements in opioid dose and risk mitigation.

Prior Presentation A preliminary form of these results was presented at the VA HSR\&D Annual Conference, Washington, D.C., July 2017; an upated report was presented at the VA HSR\&D Annual Conference, Washinaton. D.C. October 2019.

Received May 24, 2019

Revised October 4, 2019

Accepted November 21, 2019

Published online December 17, 2019
KEY WORDS: pain; opioids; veterans; interdisciplinary; primary care.

J Gen Intern Med 35(4): 1238-44

DOI: $10.1007 / \mathrm{s} 11606-019-05572-9$

(C) Society of General Internal Medicine (This is a U.S. government work and not under copyright protection in the U.S.; foreign copyright protection may apply) 2019

A s many as $50 \%$ of Veterans report chronic pain complaints and the prevalence may be as high as $75 \%$ among female veterans. ${ }^{1,2}$ Co-occurring mental health problems are especially prevalent in veterans with chronic pain, and veterans with complex chronic pain syndromes prescribed opioids are at especially high risk for misuse and adverse outcomes. ${ }^{3-}$ ${ }^{6} \mathrm{~A}$ recent landmark study demonstrated that opioids are not superior to non-opioid medications in treating chronic noncancer pain. ${ }^{7}$ Other studies have shown that opioids exacerbate mental health and chronic pain conditions, worsen psychosocial functioning, and increase risk for serious adverse events. $^{8-10}$

Chronic pain is a multidimensional experience that responds best to biopsychosocial interdisciplinary care that incorporates non-pharmacologic modalities such as psychotherapy and exercise. ${ }^{11-13}$ National guidelines have endorsed this approach, ${ }^{14-17}$ yet most patients with chronic pain still present to individual primary care providers (PCPs) who have relatively little training or support in managing complex chronic pain and mitigating opioid risk. ${ }^{18,19}$ Further, there is limited evidence that biopsychosocial approaches decrease opioid use or risk, ${ }^{20-22}$ and to our knowledge, there are no evidence-based guidelines for implementing interdisciplinary pain care within a primary care setting.

The national VA Opioid Safety Initiative (OSI), implemented in 2013, advises reduction or discontinuation of opioids for the management of chronic pain. ${ }^{23}$ OSI also promotes several opioid risk mitigation measures. Recent OSI performance metrics demonstrate significant improvements in opioid safety since OSI was implemented. ${ }^{23}$

The SFVAHCS Integrated Pain Team (IPT) Clinic was established in 2015, inspired by two other interdisciplinary pain clinics in the VA healthcare system. ${ }^{21,} 22$ IPT is based on the biopsychosocial model of pain care and consists of a 
collocated medical provider, psychologist, and pharmacist embedded in primary care. ${ }^{24}$ This quality improvement (QI) study used a prospective matched cohort design and VA administrative data to determine whether the IPT model is superior to usual primary care (UPC) in decreasing opioid dose and meeting three OSI targets: (1) increasing UDS monitoring, (2) increasing naloxone kit distribution, and (3) decreasing co-prescription of opioids and benzodiazepines. Reporting of findings are guided by Standards for Quality Improvement Reporting Excellence (SQUIRE 2.0). ${ }^{25}$

\section{METHODS}

\section{Context for the Study}

This QI study was conducted between November 2015 and January 2018 throughout the SFVAHCS, a multi-campus VA healthcare system serving over 40,000 veterans. The SFVAHCS includes the San Francisco VA Medical Center (SFVAMC), an urban campus, and six affiliated outpatient campuses serving both rural and urban areas across Northern California. A local SFVAHCS OSI committee was chartered in 2015 that focuses on reducing opioids in patients prescribed $\geq 90 \mathrm{mg}$ MEDD, encouraging UDS monitoring and naloxone kit distribution, and decreasing co-prescription of opioids and benzodiazepines.

\section{Description of the Intervention}

The Integrated Pain Team (IPT) is an interdisciplinary, collocated team of primary care providers with training in pain management and motivational interviewing and shared decision-making; pain pharmacists; and pain psychologists. IPT members are based at the SFVAMC, embedded in primary care, where visits are conducted in-person and via video telehealth for veterans living in rural areas. Other PCPs at SFVAMC and affiliated community-based clinics may refer veterans to IPT for short-term management (a median of 4 visits in 6 months; 60 min for an initial visit with 30-min follow-up visits). IPT's main goals are to improve pain and decrease opioid risk through opioid dose reduction and other opioid risk mitigation strategies. IPT engages patients in multimodal pain care planning based on patients' values and goals, emphasizing self-management strategies. IPT specifically encourages behavioral health strategies (e.g., cognitive behavioral therapy for chronic pain and mindfulness), physical modalities (e.g., acupuncture, chiropractic care, heat, and ice), and exercise (e.g., physical therapy, yoga, Tai Chi).

\section{Data Sources and Permissions}

Two main sources of VA administrative data were used: (1) the VA Stratification Tool for Opioid Risk Mitigation $(\text { STORM })^{26,27}$ and (2) the VA Corporate Data Warehouse (CDW). STORM extracts data from the VA electronic medical record accessible by VA providers at the point of care. STORM only includes veterans prescribed opioids and provides detailed information on opioid risk factors including opioid dose, substance use, and relevant mental and physical health diagnoses (e.g., depression, obstructive sleep apnea) and other co-prescribed pain medications and opioid risk mitigation strategies (e.g., UDS monitoring). The VA CDW was used to obtain baseline information on patient sociodemographics, pain severity (through Numeric Rating Scale (NRS) scores), verification of opioid prescriptions (drug name, dose converted to MEDD, dates prescribed), and health services utilization, including emergency department visits and hospitalizations. CDW data were accessed after the study ended with permission from the Institutional Review Board of the University of California, San Francisco and the San Francisco VA Health Care System Human Research Protection Program.

\section{Study Population and Development of the Matched Cohorts}

This was a prospective matched cohort design. Using STORM data, veterans entering the IPT clinic and prescribed opioids for chronic pain were matched sequentially (within 30 days) to patients with chronic pain prescribed opioids receiving UPC, (described below) on the following characteristics: age $(<55$ or $\geq 55$ ), gender (male or female), number and type of psychiatric diagnoses (PTSD, depression, bipolar disorder, and/or other psychiatric diagnosis), and baseline daily opioid dose (MEDD $<100$ or $\geq 100$ ). Patients prescribed methadone or buprenorphine/naloxone for treatment of opioid use disorder at baseline were excluded. Patients were followed for 6 months and compared at baseline, 3 months, and 6 months. The final study cohort consisted of 294 patients: 147 in the IPT group and 147 matched controls in UPC.

\section{Dependent Outcome Variables}

The primary dependent outcome variable was opioid dose, expressed as MEDD and calculated from opioid prescriptions in VA CDW, using the formula from the Opioid Oral Morphine Milligram Equivalent (MME) Conversion Factors. ${ }^{28}$ We calculated MEDD at baseline, 3 months, and 6 months, including opioid prescriptions within a 30-day window before and after each of the three time points. We included common oral and transdermal opioids in the VA formulary (e.g., codeine, fentanyl, hydrocodone, methadone, morphine, oxycodone, tramadol), but excluded opioids rarely used in outpatient care (e.g., butorphanol, levorphanol). If patients discontinued traditional opioids and initiated the partial opioid agonist/ antagonist, buprenorphine (in any formulation) during follow-up, their MEDD was zeroed as in other studies. ${ }^{29}$ Secondary outcomes included opioid risk mitigation, including UDS monitoring, receipt of a naloxone kit, and co-prescription of opioids and benzodiazepines. CDW was used to capture frequency of adverse clinical events in the past year, including emergency department visits and inpatient hospitalization at VA or non-VA facilities, opioid overdoses, suicide-related events, falls, and motor vehicle accidents. 


\section{Independent Variables}

The main independent variable was pain care approach: IPT versus UPC. In UPC, pain care is delivered during regular 30min primary care visits in which a single PCP (as opposed to an interdisciplinary team) addresses other physical and mental health problems. Unlike in IPT, pain may not be the main focus of the visit; thus, while UPC patients are subject to opioid safety initiatives, implementation may not be consistent owing to competing priorities and the lack of an interdisciplinary pain team. Other covariates were captured through VA CDW: age, sex, race/ethnicity and marital status, persistent moderate-to-severe pain (NRS pain scores of $\geq 4$ on three or more occasions for at least 3 months during the year prior to study entry), ${ }^{30}$ and duration of opioid therapy prior to study entry. We used VA STORM to identify mental health disorders (depression, anxiety, PTSD, alcohol use disorder, opioid use disorder and other drug use disorders) and chronic medical conditions (sleep apnea, chronic pulmonary disease, cognitive impairment, chronic kidney disease, and hepatic disease), which can increase risk for adverse opioid-related events. ${ }^{26,27}$

\section{Statistical Analyses}

We compared the two groups on sociodemographic variables, physical and mental health status and diagnoses, health services utilization, opioid risk mitigation, and adverse events. Continuous variables were compared using Wilcoxon signed-rank test, and categorical variables were compared using McNemar's chi-square test (2-level), Exact McNemar test (2-level, cell size $<5$ ), or symmetry test (multiple-level) for categorical variables of matched pairs. For the primary outcome, change in prescribed opioid dose, we compared between-group differences at baseline, 3 months, and 6 months. Means and standard deviations were compared using the Wilcoxon signed-rank sum test for non-normally distributed matched pairs. We compared group differences in the number of patients achieving a binary $\geq 50 \%$ opioid dose reduction (in MEDD) from baseline to 3 and 6 months using McNemar's test. Subsequently, we performed a conditional logistic regression for the binary outcome at 3 months and 6 months, adjusting for variables that were different between the two groups after matching. Finally, we fit a mixed-effects linear regression model for change in opioid dose with group $\times$ time point interaction terms, adjusting for covariates. For all statistical tests, we considered $\alpha=0.05$ as the threshold for statistical significance. Analyses were performed using SAS (SAS Institute Inc., 2013) and Stata (Stata Corp, 2015).

\section{RESULTS}

During the study period, 147 veterans in IPT were matched sequentially to 147 veterans in UPC on age, gender, mental health diagnoses, and baseline opioid dose, yielding no between-group differences in these characteristics. In the total sample of 294 veterans, mean age was 63 years ( $\mathrm{SD} \pm 12$ years); $30(10 \%)$ were women; $63 \%$ had service-connected disabilities. Mental health diagnoses were prevalent in both groups: $65 \%$ had $\geq 2$ mental health diagnoses. Veterans in both groups had received opioid therapy for an average of 14 years ( $\mathrm{SD} \pm 5.2$ years) and the mean opioid dose (in MEDD) was $124.3 \mathrm{mg}$ (SD \pm 236.3 $\mathrm{mg}$ ), exceeding thresholds for safety. Nevertheless, in the year prior to the study $<2 \%$ had experienced an opioid overdose, suicide-related event or motor vehicle accident (Table 1).

After matching, some baseline differences were noted. First, more African American and other minority groups (e.g., Asian and Native American) received pain care through IPT (25\%) versus UPC $(12 \%)(p=0.02)$. More veterans in IPT $(96 \%)$ reported persistent moderate-to-severe chronic pain than veterans in UPC $(88 \%, p=0.01)$. Similarly, more veterans in IPT (versus UPC) had diagnoses of opioid use disorder (17\% versus $6 \%, p<0.01)$ and other non-alcohol drug use disorders ( $8 \%$ versus $3 \%, p=0.05$ ). In the year prior to the study, significantly more veterans in IPT versus UPC presented to emergency departments $(60 \%$ versus $46 \%, p<0.01)$ and there was a trend toward more falls among veterans in the IPT group (10 versus 5 falls, $p=0.06$ ) (Table 1 ).

From baseline to 3 months, mean MEDD decreased from 124.1 to $82.5 \mathrm{mg}$ in the IPT group and from 124.5 to $116.4 \mathrm{mg}$ in UPC, with further reductions to $68.4 \mathrm{mg}$ (IPT) and $107.1 \mathrm{mg}$ (UPC) at 6 months. The mean opioid dose reduction was significantly greater in the IPT versus UPC group by 6 months $(p<0.03)$ (Table 2). An adjusted mixed-effects linear regression model showed that the MEDD reduction in IPT was $33.6 \mathrm{mg}(95 \%$ CI $12.2-55.0 \mathrm{mg})$ greater than in UPC at 3 months ( $p<0.01)$ and $38.2 \mathrm{mg}$ (95\% CI 13.0-63.5 mg) greater than UPC by 6 months $(p<0.01)$, independent of race/ethnicity, baseline opioid dose, pain severity, opioid use disorder, receipt of a naloxone kit, co-prescription of opioids and benzodiazepines, and greater number of emergency department visits (Table 3, Fig. 1). The model revealed that patients with higher opioid doses at baseline had significantly smaller reductions in opioid dose over time, independent of treatment group and other covariates (Table 3). Compared with UPC, significantly more patients in IPT achieved $\geq 50 \%$ reduction in opioid dose at 3 months and by 6 months (Table 4 ). In adjusted analyses, the odds of achieving $50 \%$ or more reduction in opioid dose in the IPT group were more than twice that of the UPC group at 3 and 6 months (Table 5).

There were also greater improvements in opioid risk mitigation in the IPT group compared with UPC. At both 3 and 6 months, more veterans in IPT had received UDS monitoring, despite more veterans in UPC having received UDS monitoring at baseline. Similarly, significantly more veterans in IPT received naloxone kits and training (69\% versus $21 \%$ at 3 months and $71 \%$ versus $24 \%$ at 6 months; $p$ values $<0.001$ ). From baseline to 6 months, the number of veterans co-prescribed opioids with benzodiazepines decreased from 24 to 16 in the IPT group, whereas in UPC, there was an increase from none to 7 veterans by 3 months (Table 6). Finally, while veterans in IPT 
Table 1 Baseline Sociodemographic and Clinical Characteristics of Patients by Clinic Type $(N=294)$

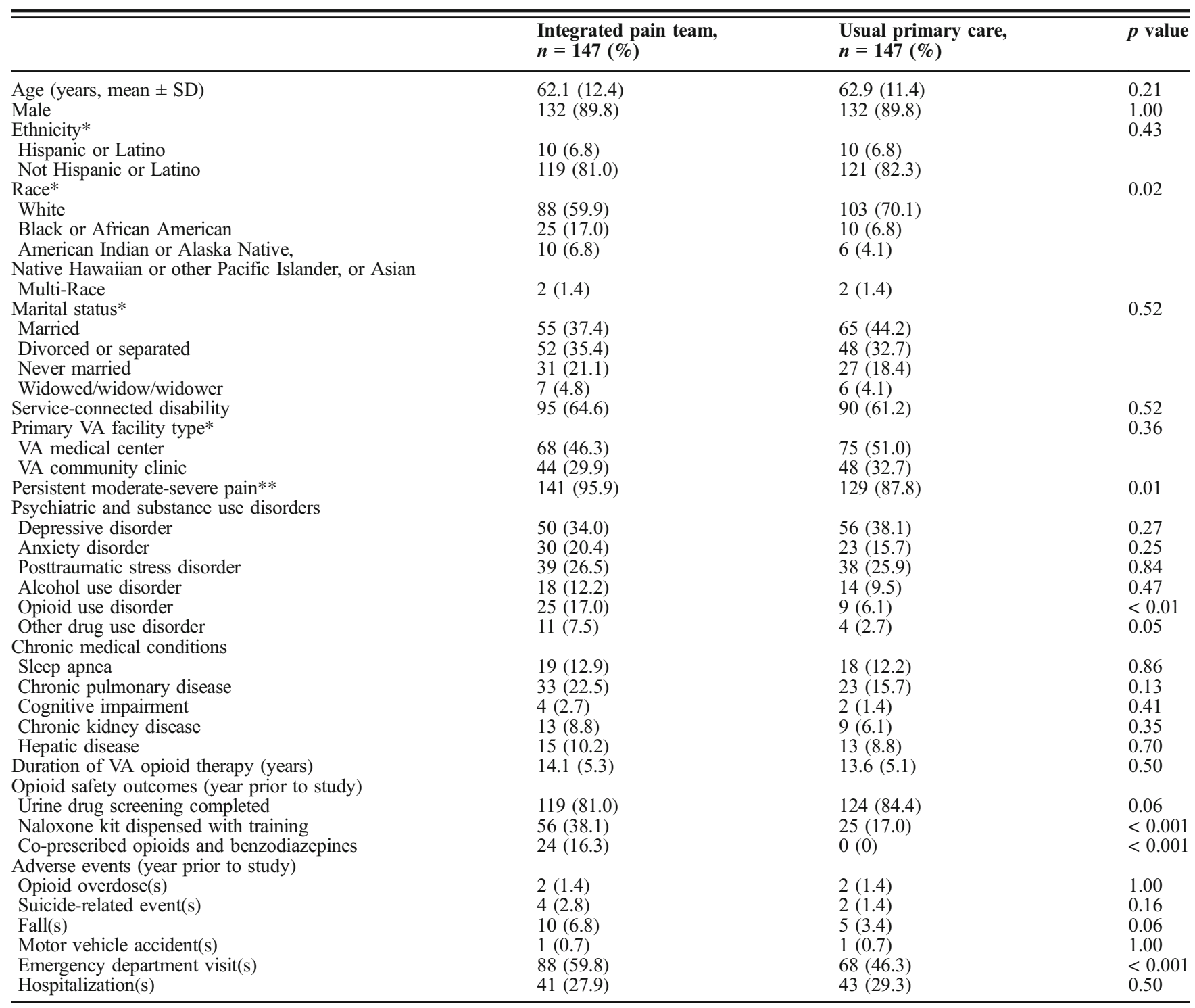

* Percentages do not sum to $100 \%$ due to missing or unknown data

$* *$ Persistent moderate-severe pain is equivalent to a pain score $>4$ and $\leq 10$ for at least 3 months

had significantly more emergency department visits in the year prior to study entry, by 6 and 12 months, the number of emergency department visits in the IPT group decreased such that this was not significantly different between groups

Table 2 Unadjusted Change in Mean Opioid Dose in Morphine Equivalent Daily Dose (MEDD in milligrams) at 3 and 6 Months by Clinic Type

\begin{tabular}{llll}
\hline \hline & $\begin{array}{l}\text { Integrated } \\
\text { pain } \\
\text { team, } \boldsymbol{n}=\mathbf{1 4 7}\end{array}$ & $\begin{array}{l}\text { Usual } \\
\text { primary } \\
\text { care, } \boldsymbol{n}=\mathbf{1 4 7}\end{array}$ & $\begin{array}{l}\boldsymbol{p} \\
\text { value }\end{array}$ \\
\hline $\begin{array}{l}\text { Baseline dose, mean } \\
\text { MEDD (SD) }\end{array}$ & $124.1(241.1)$ & $124.5(231.5)$ & 0.37 \\
$\begin{array}{l}\text { 3-month dose, mean } \\
\text { MEDD (SD) }\end{array}$ & $82.5(157.7)$ & $116.4(230.0)$ & 0.15 \\
$\begin{array}{l}\text { 6-month dose, mean } \\
\text { MEDD (SD) }\end{array}$ & $68.4(166.1)$ & $107.1(223.4)$ & 0.03 \\
\hline
\end{tabular}

( $p$ values $=0.08$ and 0.29 at 6 and 12 months respectively). Implementation of the IPT clinic is described elsewhere. ${ }^{19,31}$

Table 3 Adjusted Change in Opioid Dose (MEDD in Milligrams) by Clinic Type and Time*

\begin{tabular}{lll}
\hline \hline & Coefficient $(95 \%$ CI $)$ & $p$ value \\
\hline $\begin{array}{l}\text { Integrated pain team versus } \\
\text { usual primary care (baseline) }\end{array}$ & $1.6(-7.8$ to 11.0$)$ & 0.74 \\
Time (baseline as reference) & & \\
3 months & $-8.2(-15.6$ to -0.8$)$ & 0.03 \\
6 months & $-17.7(-29.2$ to -6.2$)$ & $<0.0$ \\
IPT* time & $-33.6(-55.0$ to -12.2$)$ & $<0.01$ \\
IPT at 3 months & $-38.2(-63.5$ to -13.0$)$ & $<0.01$ \\
IPT at 6 months &
\end{tabular}

*Adjusted for race, baseline persistent moderate-to-severe pain, opioid use disorder, baseline opioid use (MEDD in milligrams), naloxone kit distribution, co-prescription of opioids and benzodiazepines, and number of emergency department visits 


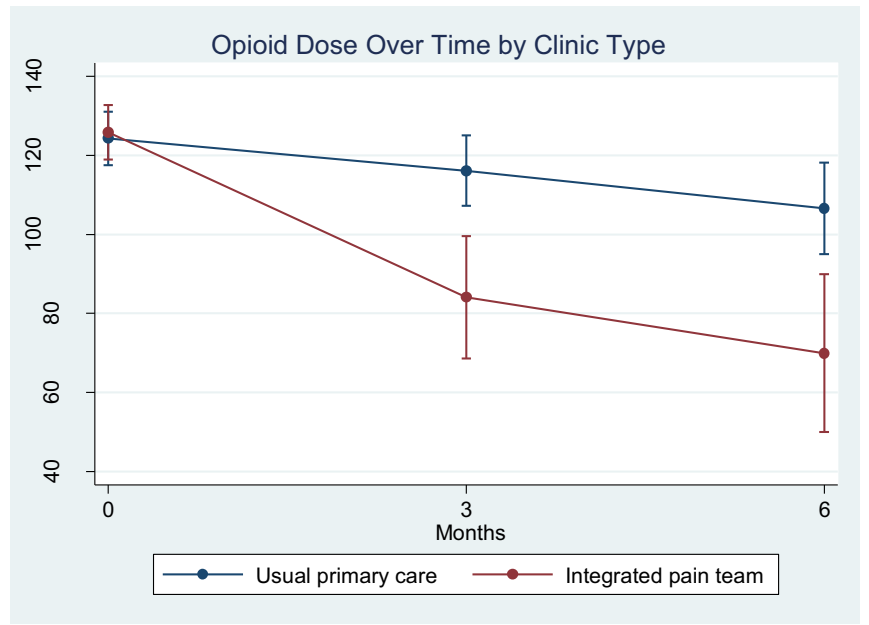

Figure 1 Change in opioid dose over time by clinic type in standardized units known as Morphine Equivalent Daily Dose (MEDD) in milligrams. Analyses were adjusted for race, baseline persistent moderate-severe pain, baseline opioid use (MEDD in milligrams), opioid use disorder, naloxone kit distribution, co-prescription of opioids and benzodiazepines, and number of emergency department visits. Veterans receiving care in the Integrated Pain Team clinic demonstrated significantly greater reductions at 3 months which, by 6 months, continued to decrease from baseline compared with veterans receiving usual primary care.

\section{DISCUSSION}

In this QI study, we examined whether the Integrated Pain Team approach was more effective than the usual primary care in reducing opioid dose and in uptake of opioid risk mitigation strategies in veterans enrolled in VA primary care. Because the two groups were matched, they were intentionally similar on key variables, but veterans referred to IPT had higher risk characteristics than their UPC counterparts. For example, veterans in IPT had a higher prevalence of moderate-to-severe pain (versus mild), diagnoses of opioid or other drug use disorders, past-year emergency department visits, and opioid-benzodiazepine co-prescription. Despite these higher risk characteristics at baseline, veterans receiving care in IPT achieved significantly greater decreases in opioid dose and improvements in risk mitigation after 3 months that continued improving up to 6 months. These results indicate that IPT is more effective than usual primary care in improving opioidrelated outcomes. Also, longer engagement with IPT is associated with greater improvements in opioid dose and uptake of risk mitigation strategies.

In IPT, the mean MEDD dropped to $<70 \mathrm{mg}$ by 6 months, whereas in the UPC group, mean MEDD never dropped below $100 \mathrm{mg}$, remaining above the threshold established by VA OSI (90 mg MEDD). ${ }^{23}$ Chronic pain and opioid use/dependence are each associated with underlying mental health conditions. ${ }^{3-5}$ In the IPT model, psychologists use cognitive

Table 4 Proportion of Veterans Reducing Opioid Dose (MEDD in Milligrams) $\geq 50 \%$ from Baseline at 3 and 6 Months by Clinic Type

\begin{tabular}{llll}
\hline \hline & $\begin{array}{l}\text { Integrated pain } \\
\text { team, } \boldsymbol{n}=\mathbf{1 4 7}\end{array}$ & $\begin{array}{l}\text { Usual primary } \\
\text { care, } \boldsymbol{n}=\mathbf{1 4 7}\end{array}$ & $\begin{array}{l}\boldsymbol{p} \\
\text { value }\end{array}$ \\
\hline $\begin{array}{l}3 \text { months, } n \\
(\%)\end{array}$ & $44(29.9)$ & $23(15.7)$ & $<0.01$ \\
$\begin{array}{l}\text { ( months, } n \\
(\%)\end{array}$ & $59(40.1)$ & $33(22.5)$ & $<0.01$ \\
\hline
\end{tabular}

behavioral therapy to modify underlying neuropsychological drivers of persistent pain, such as catastrophizing, depression and anxiety, or motivational interviewing (MI) to motivate behavioral modification surrounding opioid use. ${ }^{13,} 32,33$ The PCP in IPT also uses MI and shared decision-making to develop a multimodal pain care plan including evidencebased modalities like yoga, mindfulness, and acupuncture, ${ }^{13}$, 34,35 while the collocated pharmacist can oversee individualized opioid tapers. Also, as IPT members are collocated in one treatment room, not only is this "one-stop shop model" spaceand time-efficient but also patients and providers can be unified around treatment goals, avoiding "splitting" and fragmented care, which can thwart attempts to replace opioids with multimodal pain care alternatives. ${ }^{19,31}$

At most VAs, PCPs practice in Patient-Aligned Care Teams (PACTs) that include psychologists and pharmacists embedded in usual primary care. ${ }^{36,37}$ VA OSI makes additional services available in usual primary care with pharmacists dedicated to opioid safety monitoring and tapering. ${ }^{23}$ Nevertheless, PCPs are still limited to 30-min encounters which makes it challenging to focus on chronic pain and opioid concerns in the context of veterans' other medical problems, and despite the availability of PACT psychologists and pharmacists, it is difficult for PCPs to quickly assemble a one-stop

Table 5 Odds of Reducing Opioid Dose (MEDD in Milligrams) $\geq$ $50 \%$ from Baseline at 3 and 6 Months by Clinic Type*

\begin{tabular}{lll}
\hline \hline & Odds ratio (95\% CI) & $\boldsymbol{p}$ value \\
\hline Unadjusted & & \\
3 months: IPT versus UPC & $2.62(1.38-4.96)$ & $<0.01$ \\
6 months: IPT versus UPC & $2.53(1.44-4.43)$ & $<0.01$ \\
Adjusted* & & \\
3 months: IPT versus UPC & $2.35(1.04-5.35)$ & 0.04 \\
6 months: IPT versus UPC & $2.03(1.04-3.95)$ & 0.04 \\
\hline
\end{tabular}

*Adjusted for race, baseline opioid dose (MEDD in milligrams), persistent moderate-to-severe pain, and opioid use disorder 
Table 6 Change in Proportion of Veterans Achieving Opioid Safety Outcomes from Baseline to 3 and 6 Months by Clinic Type

\begin{tabular}{|c|c|c|c|}
\hline & $\begin{array}{l}\text { Integrated pain } \\
\text { team, } \\
n=147(\%)\end{array}$ & $\begin{array}{l}\text { Usual primary } \\
\text { care, } \\
n=147(\%)\end{array}$ & $p$ value \\
\hline \multicolumn{4}{|c|}{ Urine drug screen monitoring } \\
\hline Baseline & $119(81.0)$ & $124(84.4)$ & 0.06 \\
\hline 3 months & $131(89.1)$ & $110(74.8)$ & $<0.01$ \\
\hline 6 months & $117(80.1)$ & $106(72.6)$ & $<0.01$ \\
\hline \multicolumn{4}{|c|}{ Naloxone kit distribution } \\
\hline Baseline & $56(38.1)$ & $25(17.0)$ & $<0.001$ \\
\hline 3 months & $102(69.4)$ & $31(21.1)$ & $<0.001$ \\
\hline 6 months & $105(71.4)$ & $35(24.0)$ & $<0.001$ \\
\hline \multicolumn{4}{|c|}{ Co-prescribed opioids and benzodiazepines } \\
\hline Baseline & $24(16.3)$ & $0(0)$ & $<0.001$ \\
\hline 3 months & $16(10.9)$ & $7(4.8)$ & 0.05 \\
\hline 6 months & $16(10.9)$ & $4(2.7)$ & $<0.001$ \\
\hline
\end{tabular}

shop model with the same "teamness" as IPT. This may explain why we detected decreases in opioid dose in UPC, but IPT was significantly more effective. For solo practitioners outside of an integrated system like VA, it is even more challenging to assemble an interprofessional team that uses a common electronic medical record to support the intensive behavioral change required for a durable reduction in opioids, like that achieved by IPT. ${ }^{24}$

From a systems perspective, IPT is more staff- and timeintensive than UPC, requiring the colocation of three different interprofessional clinicians for initial and some follow-up visits. Indeed, there was a median of four visits with IPT compared with two visits for patients in UPC during the same 6-month period. It was beyond the scope of this QI project to compare costs, but it is possible that additional up-front costs incurred by IPT may be offset by subsequent reductions in higher-cost services, such as emergency department visits as evidenced in this study. Also, with IPT's focus on nonpharmacological self-management strategies, overall costs of IPT may be lower than if similar patients underwent invasive procedures or surgery, both of which have mixed outcomes for chronic pain conditions. ${ }^{16,38}$ Future studies can compare costs of IPT models with UPC and tertiary care for chronic pain management in comparable patients, considering a variety of clinical outcomes, including those most important to patients such as functioning and quality of life. ${ }^{31}$

There are several limitations to this study. This was a relatively small QI study using a non-randomized matched cohort design. Though we attempted to make veterans in IPT and UPC as comparable as possible, differences remained such that patients in IPT were higher risk than veterans receiving UPC at baseline. While a difference in this direction may have been acceptable, there may have been other systematic differences and selection biases that were not measured. For example, patients referred to and remaining in IPT care (versus UPC) may have been more motivated to achieve study outcomes. Third, we relied on VA pharmacy data which indicates that opioid medication was dispensed and how often, but not how patients were taking it. Finally, our results may not generalize to women or non-veteran populations. Curiously, more African American and other minority groups were referred to IPT, possibly because of implicit bias, but our study design did not allow us to investigate this further. ${ }^{39}$ Notably, this paper does not focus on patient-reported outcomes such as pain severity, functioning, quality of life, or satisfaction with pain care, but a forthcoming publication indicates improvements in these areas in patients in IPT.

In sum, higher risk veterans followed by IPT achieved greater opioid dose reductions and improved opioid safety at 3 and 6 months than their lower risk counterparts followed in usual primary care. This study provides compelling preliminary data to support an interdisciplinary collocated primary care-based team approach to decrease opioid prescribing and improve risk mitigation in patients with chronic pain.

Acknowledgments: The results reported herein would not be possible without the hard work of members of the San Francisco VA Health Care System Integrated Pain Team staff whose members include Erin Watson, PsyD.; Payal Mapara, PsyD; John Sorrell, PhD; Caitlin Garvey, NP; Wilson Fong, NP; Andrea Lynn, RN; Elizabeth Son, Pharm D.; and Christina Tat, Pharm D. We thank Thomas Metzler for assisting with assembling the matched cohort.

Corresponding Author: Karen H. Seal, MD, MPH; San Francisco Veterans Affairs Health Care System, University of California, San Francisco, San Francisco, CA, USA (e-mail: Karen.Seal@va.gov).

Funding Information This research is funded by a Department of Veterans Affairs Quality Enhancement Research Initiative (QUERI) award (Grant no: 15-283; Mary Whooley, MD, Principal Investigator; Karen Seal, Co-Investigator).

\section{Compliance with Ethical Standards:}

Conflict of Interest: The authors declare that they do not have a conflict of interest.

Disclaimer: The views expressed are those of the authors only and do not reflect the opinions of the Veterans Health Administration.

\section{REFERENCES}

1. Kerns RD, Otis J, Rosenberg R, Reid MC. Veterans' reports of pain and associations with ratings of health, health-risk behaviors, affective distress, and use of the healthcare system. J Rehabil Res Dev. 2003;40(5):371-379.

2. Haskell SG, Heapy A, Reid MC, Papas RK, Kerns RD. The prevalence and age-related characteristics of pain in a sample of women veterans receiving primary care. J Womens Health (Larchmt). 2006;15(7):862-869.

3. Bair MJ, Wu J, Damush TM, Sutherland JM, Kroenke K. Association of depression and anxiety alone and in combination with chronic musculoskeletal pain in primary care patients. Psychosom Med. 2008;70(8):890-897.

4. Shipherd JC, Keyes M, Jovanovic T, et al. Veterans seeking treatment for posttraumatic stress disorder: what about comorbid chronic pain? J Rehabil Res Dev. 2007;44(2):153-166.

5. Seal KH, Shi Y, Cohen G, et al. Association of mental health disorders with prescription opioids and high-risk opioid use in US veterans of Iraq and Afghanistan. JAMA. 2012;307(9):940-947.

6. Seal KH, Bertenthal D, Barnes DE, et al. Traumatic Brain Injury and Receipt of Prescription Opioid Therapy for Chronic Pain in Iraq and Afghanistan Veterans: Do Clinical Practice Guidelines Matter? J Pain. 2018;19(8):931-941 
7. Krebs EE, Gravely A, Nugent S, et al. Effect of Opioid vs Nonopioid Medications on Pain-Related Function in Patients with Chronic Back Pain or Hip or Knee Osteoarthritis Pain: The SPACE Randomized Clinical Trial. JAMA. 2018;319(9):872-882.

8. Sullivan MD, Edlund MJ, Zhang L, Unutzer J, Wells KB. Association between mental health disorders, problem drug use, and regular prescription opioid use. Arch Intern Med. 2006;166(19):2087-2093.

9. Sullivan MD, Leigh $\mathbf{J}$, Gaster B. Brief report: Training internists in shared decision making about chronic opioid treatment for noncancer pain. J Gen Intern Med. 2006;21(4):360-362.

10. Harris KM, Edlund MJ. Self-medication of mental health problems: new evidence from a national survey. Health Serv Res. 2005;40(1):117-134.

11. Cheatle MD. Biopsychosocial Approach to Assessing and Managing Patients with Chronic Pain. Med Clin North Am. 2016;100(1):43-53.

12. Gatchel RJ, McGeary DD, McGeary CA, Lippe B. Interdisciplinary chronic pain management: past, present, and future. Am Psychol. 2014;69(2):119-130.

13. Kligler B, Bair MJ, Banerjea R, et al. Clinical Policy Recommendations from the VHA State-of-the-Art Conference on Non-Pharmacological Approaches to Chronic Musculoskeletal Pain. J Gen Intern Med. 2018;33(Suppl 1):16-23.

14. Institute of Medicine Committee on Advancing Pain Research, Care and Education. National Pain Strategy: A Comprehensive Population HealthLevel Strategy for Pain. 2016.

15. Dowell D, Haegerich TM, Chou R. CDC Guideline for Prescribing Opioids for Chronic Pain-United States, 2016. JAMA. 2016;315(15):1624-1645.

16. Institute of Medicine Committee on Advancing Pain Research $\mathrm{C}$, and Education. Relieving Pain in America: A Blueprint for Transforming Prevention, Care, Education, and Research. Washington (DC): National Academies Press (US);2011.

17. The Opioid Therapy for Chronic Pain Work Group; Veterans Health Administration and Department of Defense. VA/DoD Clinical Practice Guideline for Opioid Therapy for Chronic Pain. Version 3.0. In: Defense VHAaDo, ed. Washington, DC; 2017.

18. Jamison RN, Sheehan KA, Scanlan E, Matthews M, Ross EL. Beliefs and attitudes about opioid prescribing and chronic pain management: survey of primary care providers. J Opioid Manag. 2014;10(6):375-382.

19. Purcell N, Zamora K, Tighe J, Li Y, Douraghi M, Seal K. The Integrated Pain Team: A Mixed-Methods Evaluation of the Impact of an Embedded Interdisciplinary Pain Care Intervention on Primary Care Team Satisfaction, Confidence, and Perceptions of Care Effectiveness. Pain Med 2018; 19(9):1748-1763.

20. Chou R, Turner JA, Devine EB, et al. The effectiveness and risks of long-term opioid therapy for chronic pain: a systematic review for a National Institutes of Health Pathways to Prevention Workshop. Ann Intern Med. 2015; 162(4):276-286.

21. Becker WC, Edmond SN, Cervone DJ, et al. Evaluation of an Integrated, Multidisciplinary Program to Address Unsafe Use of Opioids Prescribed for Pain. Pain Med. 2018;19(7):1419-1424.

22. Wiedemer NL, Harden PS, Arndt IO, Gallagher RM. The opioid renewal clinic: a primary care, managed approach to opioid therapy in chronic pain patients at risk for substance abuse. Pain Med. 2007;8(7):573-584.

23. Gellad WF, Good CB, Shulkin DJ. Addressing the Opioid Epidemic in the United States: Lessons From the Department of Veterans Affairs. JAMA Intern Med. 2017; 177(5):611-612.

24. Seal K, Becker W, Tighe J, Li Y, Rife T. Managing Chronic Pain in Primary Care: It Really Does Take a Village. J Gen Intern Med. 2017;32(8):931-934
25. Ogrinc G DL, Goodman D, Batalden PB, Davidoff F, Stevens D. SQUIRE 2.0 (Standards for QUality Improvement Reporting Excellence): Revised publication guidelines from a detailed consensus process. BMJ Qual Saf.

26. Minegishi T, Frakt AB, Garrido MM, et al. Randomized program evaluation of the Veterans Health Administration Stratification Tool for Opioid Risk Mitigation (STORM): A research and clinical operations partnership to examine effectiveness. Subst Abus. 2019;1-6.

27. Oliva EM, Bowe T, Tavakoli S, et al. Development and applications of the Veterans Health Administration's Stratification Tool for Opioid Risk Mitigation (STORM) to improve opioid safety and prevent overdose and suicide. Psychol Serv. 2017;14(1):34-49.

28. National Center for Injury Prevention and Control. Centers for Disease Control and Prevention. CDC compilation of benzodiazepines, muscle relaxants, stimulants, zolpidem, and opioid analgesics with oral morphine milligram equivalent conversion factors. Atlanta, GA; 2016.

29. Oldfield BJ, Edens EL, Agnoli A, et al. Multimodal Treatment Options, Including Rotating to Buprenorphine, Within a Multidisciplinary Pain Clinic for Patients on Risky Opioid Regimens: A Quality Improvement Study. Pain Med. 2018;19(suppl_1):S38-S45.

30. Haskell SG, Brandt CA, Krebs EE, Skanderson M, Kerns RD, Goulet JL. Pain among Veterans of Operations Enduring Freedom and Iraqi Freedom: do women and men differ? Pain Med. 2009;10(7):1167-1173.

31. Purcell N, Zamora $\mathbf{K}$, Gibson $\mathbf{C}$, et al. Patient experiences with integrated pain care: A qualitative evaluation of one VA's biopsychosocial approach to chronic pain treatment and opioid safety. Glob Adv Health Med. 2019;8:2164956119838845.

32. Ehde DM, Dillworth TM, Turner JA. Cognitive-behavioral therapy for individuals with chronic pain: efficacy, innovations, and directions for research. Am Psychol. 2014;69(2):153-166.

33. Alperstein D, Sharpe L. The Efficacy of Motivational Interviewing in Adults With Chronic Pain: A Meta-Analysis and Systematic Review. J Pain. 2016;17(4):393-403.

34. Kerns RD, Krebs EE, Atkins D. Making Integrated Multimodal Pain Care a Reality: A Path Forward. J Gen Intern Med. 2018;33(Suppl 1):1-3.

35. Giannitrapani K, Holliday J, Miake-Lye I, Hempel S, Taylor SL. Synthesizing the Strength of the Evidence of Complementary and Integrative Health Therapies for Pain. Pain Med. 2019.

36. Yano EM, Bair MJ, Carrasquillo O, Krein SL, Rubenstein LV. Patient Aligned Care Teams (PACT): VA's journey to implement patient-centered medical homes. J Gen Intern Med. 2014;29 Suppl 2:S547-549.

37. Gilman SC, Chokshi DA, Bowen JL, Rugen KW, Cox M. Connecting the dots: interprofessional health education and delivery system redesign at the Veterans Health Administration. Acad Med: J Assoc Am Med Coll. 2014;89(8):1113-1116.

38. Gatchel RJ, Peng YB, Peters ML, Fuchs PN, Turk DC. The biopsychosocial approach to chronic pain: scientific advances and future directions. Psychol Bull. 2007;133(4):581-624.

39. Pletcher MJ, Kertesz SG, Kohn MA, Gonzales R. Trends in opioid prescribing by race/ethnicity for patients seeking care in US emergency departments. JAMA. 2008;299(1):70-78.

Publisher's Note Springer Nature remains neutral with regard to jurisdictional claims in published maps and institutional affiliations. 